\title{
Competencias matemáticas como factor de éxito en la prueba pro en universidades de Barranquilla, Colombia
}

\section{Mathematical competencies as a success factor for the Saber Pro Test at the Universities of Barranquilla, Colombia}

\author{
Plutarco Segundo Martínez-Bustos \\ Universidad de Sucre, Sincelejo, Colombia \\ William Alejandro Niebles-Núñez \\ Universidad de Sucre, Sincelejo, Colombia \\ Y Universidad del Atlántico, Barranquilla, Colombia \\ Leonardo David Niebles-Núñez iD \\ Universidad del Atlántico, Barranquilla, Colombia
}

\begin{abstract}
Resumen
Objetivo: Analizar las competencias matemáticas como factor de éxito para la realización de la Prueba Saber Pro en Universidades de Barranquilla, Colombia. Se asumieron los postulados de Albrecht, Fernández (2010), Martínez (2009), Méndez (2013) y Tobón (2013), entre otros teóricos con igual relevancia, así como diferentes documentos publicados por el Ministerio de Educación Nacional. Método: Se asumió el paradigma pospositivista, cuya expresión metodológica es la cuantitativa, con un diseño no experimental, de campo y un alcance descriptivo. Los datos fueron obtenidos a través de un cuestionario, el cual fue aplicado a 244 estudiantes universitarios que cursaban los dos últimos semestres de su carrera. Resultados: Se infiere que las estrategias de enseñanza aplicada para promover los indicadores señalados como habilidades a ser aprendidas en el fortalecimiento de las competencias en matemáticas de los estudiantes universitarios son apropiadas para sus vidas académicas y profesionales. Discusión y Conclusiones: La población seleccionada evidencia un equilibrio ubicado en el rango de "favorable", lo que coadyuvaría a alcanzar los objetivos previstos.
\end{abstract}

Palabras clave: Competencias matemáticas, competencias genéricas, razonamiento cuantitativo.

\begin{abstract}
Objective: to analyze the mathematical competences as a success factor for the realization of the Pro-universities test of Barranquilla Colombia. Therefore, the postulates of Albrecht, Fernández (2010), Martínez (2009), Méndez (2013), Tobón (2013), among other theorists with equal relevance, as well as different documents published by the Colombian Ministry of Education were assumed. Method: The postpositivist paradigm was assumed, whose methodological expression is the quantitative one, with a non-experimental, field design and a descriptive scope. The data were obtained through a questionnaire, which was applied to 244 university students in the last two semesters of their careers. Results: It is inferred that applied teaching strategies to promote the indicators indicated as skills to be learned in the strengthening of mathematical competencies of university students are appropriate for their academic and professional lives. Discussion y Conclusion: The selected population evidences a balance located in the "favorable" range, which would contribute to reach the foreseen objectives.

Keywords: Mathematical Competencies, Generic Competencies, Quantitative Reasoning.
\end{abstract}

Open Access:

ISSN: $0124-2121$ E-ISSN: $2665-2420$

ARTÍCULO RSULTADO DE INVESTIGACIÓN

Copyright $\odot 2020$ By Educación y Humanismo

Editor: Patricia Martínez Barrios Universidad Simón Bolivar

\section{Correspondencia:}

William Niebles

william.niebles@unisucre.edu.co

Recibido: 04-09-2019 Aceptado: 28-10-2019 En línea desde: 11-12-2019 


\section{Introducción}

Los países preocupados por el desarrollo integral de sus ciudadanos han de promover políticas públicas orientadas a mejorar el nivel educativo de la población. Es por ello que algunos de esos países han fortalecido la educación como sector esencial para el desarrollo económico y el bienestar general.

Los países deben apostar, efectivamente, en la formación de su población invirtiendo en educación, tecnología, desarrollo humano e investigación, para así salir del atraso económico, pues esto conlleva a superar la pobreza por medio de la optimización del talento humano. Por tanto, cada vez que se avanza en la idea de un mejor desarrollo económico, se proporcionan oportunidades a los jóvenes; pero todo esto solo puede darse cuando el proceso de formación se relaciona con otros saberes delimitados en una sociedad (Aguilar, 2017, p 359).

En ese orden de ideas, en Latinoamérica han surgido procesos de transformación socioeconómicos, que son consecuencia de la dinámica histórica y de criterios e interrelaciones humanas que abarcan factores políticos, económicos, culturales y sociales que propician el diseño y el desarrollo de modelos educativos. Muchos de estos modelos se fundamentan en las propuestas de la Organización de la Naciones Unidas para el Desarrollo de la Educación, la Cultura y la Ciencia (UNESCO), mediante la educación por competencias.

Aparte de lo dicho, en las últimas décadas, tanto en el ámbito mundial como en Colombia, el sistema educativo ha estado influenciado por diversas tendencias pedagógicas, generadas por los cambios vertiginosos en la comunicación e información, los cuales incidieron en la divulgación de los avances científicos, tecnológicos, económicos, entre otros; de tal manera que se precisa asumir esta realidad, así como gestionar las significativas transformaciones.

En consonancia con lo expuesto hasta ahora, el Estado colombiano ha asumido el sistema educativo en todos sus niveles, como herramienta para fortalecer el conocimiento tecnológico, científico y humanístico. Y ello contribuye al desarrollo del potencial creativo de cada persona, promueve su participación activa, consciente y solidaria en la transformación social, concatenada con valores como la identidad nacional.

Ahora bien, el aprendizaje por competencias constituye un enfoque educativo idóneo, ya que orienta los procesos de enseñanza hacia la integración de "distintos tipos de aprendizajes (conocimientos, habilidades y actitudes) para afrontar situaciones y problemas en contextos determinados". Por consiguiente, articula diferentes perfiles, filosofías, planificación, estrategias didácticas, epistemologías y mecanismos para la evaluación; ésta implica superar la adquisición memorística de conocimiento inaplicable en la vida real para el discente. Por otra parte, las competencias corresponden a aprendizajes más globales y prácticos, dirigidos al desarrollo de la capacidad de actuación del sujeto.

Más concretamente, el desarrollo en competencias en matemáticas constituye un importante reto, pues es imperativo que se les proporcione a los estudiantes las herramientas para apropiarse del conocimiento, así como la información, con la intención de generar 
habilidades, destrezas y hábitos para ser implementados en la resolución de situaciones problemas, no solo del ámbito académico, sino también en otros contextos (Albrecht, Jiménez \& Jiménez, 2014). Sin embargo, "el panorama muestra serias dificultades en el proceso de desarrollo de competencias en las instituciones educativas que ponen de manifiesto ciertas problemática". (p.18)

A este respecto, instituciones del Estado colombiano precisan que:

Como parte de esta labor, en el año 2008 el Ministerio convocó a reconocidos miembros de la comunidad académica con el fin de propiciar la reflexión en torno al conjunto de competencias genéricas que se ajusten a las características y necesidades de nuestro contexto social y cultural, pero también al marco más amplio del panorama internacional. Como resultado de estas reflexiones, el colectivo académico convocado identificó cuatro competencias genéricas para la educación superior: Comunicación en Lengua Materna y otra Lengua internacional; Pensamiento Matemático; Ciudadanía; y Ciencia, Tecnología y Manejo de la Información (Ministerio de Educación Nacional, 2009, p. 1).

Mediante el citado documento, con la enunciación de las competencias genéricas señaladas se buscó crear indicadores de calidad para las universidades, estableciéndose estos como factor vital para su acreditación. Ello se materializa en el desempeño laboral de los egresados y se traduce en mejores oportunidades, ya que demuestran el saber, saber hacer en contexto, ser y convivir. Lo anterior induce un esfuerzo significativo en los estudiantes por adelantar las pruebas Saber de calidad (Aristizabal, 2012, p.4).

Cabe subrayar que antes del año 2009 a los estudiantes de pregrado se les aplicaban exámenes promovidos por el Estado colombiano, los cuales eran conocidos por las siglas ECAES, Exámenes de Calidad de Educación Superior-. Posteriormente, el Ministerio de Educación Nacional de Colombia (MEN), junto al Instituto Colombiano para la Evaluación de la Educación (ICFES) mediante Decreto 3963, las modifican y reglamentan, denominándolas como exámenes SABER PRO (Instituto Colombiano para la Evaluación de la Educación, 2013a).

Dichas evaluaciones, a diferencia de las primeras que se realizaban en función de objetivos, evalúan competencias básicas descritas por el Instituto Colombiano para la Evaluación de la Educación (2013b) como "aquellas que es indispensable desarrollar independientemente de la especificidad que pueda presentarse en diferentes instituciones educativas" (p. 8), las mismas buscan medir cuantitativamente a los estudiantes en su proceso formativo.

Esta valoración cuantitativa permite conocer la situación del país en materia educativa y tomar en consecuencia los correctivos donde se hallen falencias del sistema educativo. Las pruebas Saber Pro poseen un razonamiento cuantitativo, componente genérico que ayuda a reforzar el pensamiento lógico, en tanto elemento fundamental para el desarrollo del estudiante en toda su vida. Este es fortalecido en la educación universitaria con su plan de estudio cuando aborda cursos de matemáticas y estadística.

En consecuencia, para un buen desarrollo en competencias es necesario un modelo educativo cuyo empeño estén enfocado en una formación académica del estudiante, concebida esta desde su contexto social; de manera tal que las distintas disciplinas posibiliten 
generar conocimiento tomando en cuenta la cotidianidad.

Se requiere, en específico, un modelo educativo que enfoque los problemas desde distintos puntos de vista, así como con la contribución de otras disciplinas. De esta manera, "al combinar una visión holística de los problemas y una heurística para su abordaje, se hace posible proponer soluciones creativas y novedosas" (García, 2014a, p. 2).

Es evidente que, en el marco holístico-heurístico, las habilidades de enseñanza deben fortalecer las competencias. En el caso de las matemáticas, según García (2014b, p. 2), ello "implica potencialidad de abstraer, determinar regularidades, y generalizar para el desarrollo de propuestas y obtener soluciones eficientes y económicas a los problemas", teniendo en cuenta el cambio, tanto en el entorno natural como social.

En este sentido, el docente debe poseer un amplio conocimiento sobre las estrategias de enseñanza, saber cómo funcionan y como pueden utilizarse o desarrollarse en el aula. Y estas deben complementarse con las estrategias, definidas por Poggioli (2005) como "todas aquellas actividades y procedimientos que se realizan con el propósito de crear, desarrollar y mantener un ambiente apropiado para el aprendizaje y el estudio" (p. 9), así como el trabajo cooperativo, para enriquecer el proceso de enseñanza.

A la luz de lo expresado hasta ahora, en esta investigación se propone analizar las competencias matemáticas como factor de éxito para la realización de la Prueba Pro en las universidades de Colombia, con el interés de generar estrategias que contribuyan al logro del señalado objetivo, lo cual coadyuvaría al fortalecimiento académico y a la consecución de becas para estudios posgraduales.

\section{Fundamento Teórico}

\section{Competencias matemáticas}

Según Guzmán, Obonaga y Gutiérrez (2015), las competencias matemáticas facilitan "la interrelación de componentes cognitivos, procedimentales y actitudinales, que ayudan a los estudiantes a dar respuesta a los problemas a los que se enfrenten" (p. 2). Asimismo, estos autores plantean que la magnitud de dicha competencia en las personas se revela cuando ellas se enfrentan a un evento difícil, ya que enfatizan "más en lo que el estudiante puede hacer con sus conocimientos y habilidades, que en el dominio de los mismos".

Para teóricos como Tobón (2013), estas competencias básicas implican incorporar los contenidos numéricos, los cuales, según el Ministerio de Educación Nacional de Colombia (2005a, p. 48), son expresados en los Lineamientos Curriculares de Matemáticas. Ellos, en efecto, prevén una consideración pragmática e instrumental del conocimiento matemático, en la cual se utilizan los conceptos, proposiciones, sistemas y estructuras matemáticas como herramientas eficaces, mediante las cuales se llevan a la práctica determinados tipos de pensamiento lógico y matemático dentro y fuera de las organizaciones de educación.

Cabe destacar que las competencias matemáticas constituyen poderosos precursores del discurso sobre las competencias, enfocados estos en la teoría del aprendizaje significativo 
de Ausubel, Novak y Gowin (1983). Esta teoría explica como la significatividad del aprendizaje trasciende el sentido personal de lo aprendido para incidir en experiencias sociales con eficacia, sentido y utilidad. Para ello se requiere la comprensión, profunda y consolidada, que, a su vez, está relacionada con los desempeños en actividades, tareas y proyectos.

La comprensión se proyecta más allá del entendimiento de contenidos y sus redes conceptuales derivadas, pues implica aspectos vinculados con los procedimientos, técnicas, formas de opinar, así como transmitir lo comprendido e implementar de manera cotidiana en los ámbitos profesional o científico-técnico, eventos en los cuales el estudiante necesita el dominio del conocimiento adquirido en cuanto a números, su procesamiento, análisis, nociones geométricas, relaciones numéricas y resolución de problemas.

Por consiguiente, es necesario que todas estas capacidades se articulen con una dimensión amplia de la competencia, la cual según el Ministerio de Educación de Colombia (1998b), es el conjunto de conocimientos, habilidades, actitudes, comprensiones y disposiciones cognitivas, socioafectivas y psicomotoras apropiadamente relacionadas entre sí para facilitar el desempeño flexible, eficaz y con sentido de una actividad en contextos relativamente nuevos y retadores.

\section{Competencias genéricas matemáticas}

Según Bambozzi y Vadori (2009), las competencias genéricas, también conocidas como básicas, son "aquellas que se entienden como necesarias para cualquier ámbito de estudio y, generalmente, están vinculadas a la resolución de problemas, compresión y producción de textos, estrategias de aprendizaje" (p. 20), entre muchas otras cualidades atribuibles a las mismas.

Por otra parte, la Organización para la Cooperación y el Desarrollo (OECD, 2005), en su proyecto denominado Definición y Selección de Competencias (DeSeCo), define las competencias como la capacidad para responder a "demandas complejas y llevar a cabo tareas diversas de forma adecuada. Supone una combinación de habilidades prácticas, conocimientos, motivación, valores éticos, actitudes, emociones y otros componentes sociales y de comportamiento que se movilizan conjuntamente para lograr una acción eficaz." (p. 8)

Pero, estas conceptualizaciones trascienden cuando se hace referencia a las competencias matemáticas y su aplicabilidad en la cotidianidad laboral, educativa e individual.

Ello se puede constatar en el concepto expresado por Córdova y Oliveros (2014):

La competencia matemática consiste en la habilidad para utilizar, relacionar, aplicar, analizar y modelar elementos matemáticos tales como: elementos geométricos, números, símbolos, funciones, expresiones algebraicas con sus operaciones básicas, formas de expresión y razonamiento matemático, tanto para producir e interpretar distintos tipos de información, como para ampliar el conocimiento sobre aspectos cuantitativos y espaciales de la realidad, y para resolver problemas relacionados con la vida cotidiana y con el mundo laboral (p. 58).

Otra definición relevante para la investigación es la presentada por Guzmán, Obonaga y 
Gutiérrez (2015). Los citados autores interpretan las competencias matemáticas como procesos matemáticos, entendiendo que el dominio de los mismos permite a las personas "la interrelación de componentes cognitivos, procedimentales y actitudinales, que ayudan a los estudiantes a dar respuesta a los problemas a los que se enfrenten" (p. 2). A su juicio, "se enfatiza más en lo que el estudiante puede hacer con sus conocimientos y habilidades, que en el dominio de los mismos".

El Ministerio de Educación y Ciencia de España, en el Documento de trabajo "Currículo y competencias básicas", explica que la competencia matemática es:

La habilidad para utilizar y relacionar los números, sus operaciones básicas, los símbolos y las formas de expresión y razonamiento matemático, tanto para producir e interpretar distintos tipos de información, como para ampliar el conocimiento sobre aspectos cuantitativos y espaciales de la realidad, y para resolver problemas relacionados con la vida cotidiana y con el mundo laboral (Gutiérrez, Martínez, Ezequiel \& Nebreda, 2008, p. 10).

A la luz de lo expresado hasta ahora, las competencias matemáticas, per se, son genéricas, ya que, coincidiendo con lo expuesto por la institución y autores citados, forman en el individuo las habilidades y destrezas para solucionar problemas identificables tanto en la vida académica, como laboral e individual.

Por su parte, Tobón (2013), las describe como un aprendizaje significativo y comprensivo, que capacita al estudiante para resolver dificultades en cualquier situación del contexto social donde se desempeña. En nuestra consideración, dichas competencias están formadas por conocimientos previos de los estudiantes, pues las mismas no se obtienen de manera espontánea, sino que requieren de espacios de aprendizaje perfeccionados por las experiencias, así como necesidades sentidas, las cuales puedan avanzar a niveles de competencia complejos en el contexto de estudio.

Asimismo, la idea de competencia genérica ha sido objeto de interés en varias investigaciones y reflexiones que lleva la comunidad de investigadores en educación matemática, además de condicionada por la cultura y por su historia, en la cual se utilizan distintos recursos lingüísticos y expresivos, los cuales deben ser aprendidos.

Cabe destacar, que la competencia matemática deriva en lenguaje matemático necesario para el ser humano en el establecimiento de procesos de comunicación con otras personas, esto es, según Martínez (2009), para entenderse, explicar sus relaciones y puntos de vista. Conviene, entonces, poder conocer los números, las nociones geométricas, las relaciones numéricas y la resolución de problemas, para ello, es imprescindible entender, así como distinguir los signos utilizados en la matemática. Por tanto, el estudiante debe adquirir una fluidez verbal en el área de la matemática, entendida ésta como la aptitud de usar las palabras en forma correcta, ya sea de manera oral o escrita, para divulgar las ideas o pensamientos.

Según Méndez (2013), el lenguaje matemático es una forma de comunicación a través de símbolos especiales para realizar cálculos matemáticos; así, para aprender y entender las 
matemáticas hace falta conocer su idioma, sus palabras, sus signos de manera que se pueda interpretar y, más importante aún, entender los contenidos matemáticos, comprender los objetos presentes el lenguaje matemático en cualquier situación del contexto laboral o social.

\section{Componentes de las competencias matemáticas}

Las competencias matemáticas genéricas están conformadas por los siguientes factores:

- Conocimientos numéricos: según Bruno (2000, p. 54), el concepto de conocimientos numéricos aparece en los últimos años del siglo XX y apuntó hacia el fortalecimiento en los estudiantes de su conocimiento numérico como una herramienta "útil para la vida diaria". Por consiguiente, el "énfasis en la enseñanza numérica se ha puesto más en las aplicaciones y menos en la práctica rutinaria de algoritmos". Consecuentemente, este se ha constituido en un producto de los procesos mentales mediante la actividad intelectual, que implica la contextualización y sistematización del conocimiento matemático. Este pensamiento se desarrolla cuando se conoce el origen, así como el desarrollo de los conceptos, axiomas y teorías matemáticas.

Según, el Ministerio de Educación de Colombia (1998), del mismo derivan dos tipos básicos:

Tabla 1

Tipos conocimiento numérico

Tipo

Conceptual

Procedimental

\section{Definición}

Está más cercano a la reflexión y se caracteriza por ser un conocimiento teórico, producido por la actividad cognitiva, muy rico en relaciones entre sus componentes y con otros conocimientos; tiene un carácter declarativo y se asocia con el saber qué y el saber por qué.

Se relaciona con la acción, técnicas y estrategias, para representar conceptos, al igual que transformar dichas representaciones en habilidades, así como las destrezas para elaborar, comparar y ejercitar algoritmos, en argumentar convincentemente mediante la producción de mensajes numéricos, aplicar cálculos numéricos y porcentuales. El conocimiento procedimental ayuda a la construcción, además, del refinamiento del conocimiento conceptual. Por tanto, permite el uso eficaz, así como flexible en el contexto de conceptos, proposiciones, teorías y modelos matemáticos; está asociado con el saber cómo.

Fuente: Elaborado por los autores de este texto (2019).

Nota: Análisis realizado a partir de los datos recopilados en la fuente secundaria, 
- Producción de mensajes numéricos: este componente se materializa dentro del concepto de competencia matemática, la cual consiste en la gestión de conocimientos matemáticos:

En situaciones reales o simuladas de la vida cotidiana; elaborar la información a través de herramientas matemáticas (mapas, gráficos...) para poder interpretar, y poner en práctica procesos de razonamiento que conduzcan a la solución de problemas o la obtención de la información. De ello se desprende como la habilidad de analizar, interpretar y expresar con claridad y precisión informaciones, datos y argumentaciones forma parte de la competencia matemática (Villalonga, 2017, p. 9).

En ese orden de ideas, la producción de mensajes numéricos es, además de una competencia matemática, un propósito. De acuerdo con Martínez (2009), la comunicación entre individuos se enriquece por el lenguaje matemático. Los números, la geometría, la estadística y la probabilidad, por ejemplo, proporcionan a las personas de diferentes culturas las habilidades para entenderse y comprender la cotidianidad, las cuales son funcionales en la toma de decisiones según los ámbitos. Por consiguiente, esta competencia genérica dota de destrezas al profesional universitario que le posibilitan participar en su contexto social.

Citando a Tobón (2013), las matemáticas pueden constituirse, refinarse y comunicarse, por medio de diferentes lenguajes, a través de los cuales se expresan, representan, leen, escriben, hablan y escuchan.

- $\quad$ Nociones geométricas: todo lo referente a los conceptos creadores de las competencias matemáticas son recibidos desde los primeros años de educación, y las nociones geométricas no escapan a este hecho. De acuerdo con autores como Bogisic, Bressan y Crego (2005), introducir nociones geométricas en la formación académica de una persona busca formar en el estudiante habilidades y destrezas para "controlar sus relaciones con el espacio, representar y describir en forma racional el mundo que lo rodea y estudiar los entes geométrico como modelizaciones de esa realidad" (p. 87).

Por consiguiente, las nociones geométricas básicas como competencia dotan al estudiante de un sistema de representación espacial que le habilita para interpretar, entender, realizar y comunicar información correspondiente al espacio físico. Ello incide en sus habilidades para: orientación y representación espacial; el razonamiento numérico y proporcional apoyado en el uso de gráficas; realización de planos, mapas o diagramas; destrezas para la construcción, entre muchas otras capacidades ligadas a diversas profesiones, además de actividades propias del quehacer humano.

\section{Razonamiento cuantitativo}

Se entiende por razonamiento cuantitativo a un conjunto de acciones dirigidas a la solución de problemas en un ambiente determinado. Vergara, Fontalvo, Muñoz y Valbuena (2015) explican que este:

Genera todo un marco de acciones que va en aras de resolver un problema y una situación, pero que en su debida intención busca interactuar en el fortalecimiento con la razón, con el mismo conocimiento para llegar a buscar más dinámicas de compresión 
y habilidad en el individuo, de tal manera que genere un profesional integral con gran aporte a la sociedad y a la misma academia. (p. 72).

Ciertamente, el razonamiento está vinculado a la construcción apropiada de conceptos, como un requerimiento para tomar decisiones y solución de problemas, ya que se trata de mantener la rigurosidad argumentativa avalada por las demostraciones correspondientes. Es por ello que, según Íñiguez (2015), contribuyen a "descubrir las ideas básicas en una línea argumental y concebir formal e informalmente argumentos matemáticos y transformar argumentos heurísticos en demostraciones válidas" (p. 119).

El razonamiento cuantitativo representa las competencias afines con la capacidad de orientar la comprensión de conceptos matemáticos, que facilitan analizar, modelar y resolver problemas aplicando métodos y procedimientos cuantitativos y esquemáticos, ICFES (2015, pp. 3-4). Los tópicos que este aborda se pueden resumir en las siguientes dimensiones:

Tabla 2

Dimensiones razonamiento cuantitativo

\begin{tabular}{|c|c|c|}
\hline Dimensión & Definición & Competencia adquirida \\
\hline $\begin{array}{l}\text { Interpretación y } \\
\text { representación }\end{array}$ & $\begin{array}{l}\text { Es la necesidad de comprender y manipular } \\
\text { representaciones de datos cuantitativos o } \\
\text { de objetos matemáticos, en distintos } \\
\text { formatos (textos, tablas, gráficos, } \\
\text { diagramas, esquemas). Involucra, entre } \\
\text { otras cosas: extraer información local (e. g. } \\
\text { la lectura del valor asociado a determinado } \\
\text { elemento en una tabla o la identificación de } \\
\text { un punto en el gráfico de una función) o } \\
\text { global (e. g. la identificación de un } \\
\text { promedio, tendencia o patrón); comparar } \\
\text { representaciones desde una perspectiva } \\
\text { comunicativa (e. g. qué figura representa } \\
\text { algo de una forma más clara o adecuada); } \\
\text { representar de manera gráfica; y tabular } \\
\text { funciones y relaciones. }\end{array}$ & $\begin{array}{l}\text {...el evaluado comprende y } \\
\text { transforma información } \\
\text { cuantitativa presentada en } \\
\text { distintos formatos, como } \\
\text { series, gráficas, tablas y } \\
\text { esquemas. }\end{array}$ \\
\hline $\begin{array}{l}\text { Formulación } \\
y \\
\text { ejecución }\end{array}$ & $\begin{array}{l}\text { Es la capacidad de establecer, ejecutar y } \\
\text { evaluar estrategias para analizar o resolver } \\
\text { problemas que involucren información } \\
\text { cuantitativa y objetos matemáticos. } \\
\text { Involucra, entre otras cosas: modelar de } \\
\text { forma abstracta situaciones concretas, } \\
\text { analizar los supuestos de un modelo y } \\
\text { evaluar su utilidad, seleccionar y ejecutar } \\
\text { procedimientos matemáticos como } \\
\text { manipulaciones algebraicas y cálculos, } \\
\text { evaluar el resultado de un procedimiento } \\
\text { matemático. }\end{array}$ & $\begin{array}{l}\text {---el evaluado, frente a un } \\
\text { problema que involucra } \\
\text { información cuantitativa u } \\
\text { objetos matemáticos, } \\
\text { diseña planes para } \\
\text { solucionarlo, ejecuta } \\
\text { planes de solución, y } \\
\text { alcanza soluciones } \\
\text { adecuadas. }\end{array}$ \\
\hline Argumentación & $\begin{array}{l}\text { Es la capacidad de justificar o dar razón de } \\
\text { afirmaciones o juicios a propósito de } \\
\text { situaciones que involucren información } \\
\text { cuantitativa u objetos matemáticos (las } \\
\text { afirmaciones y los juicios pueden referirse a } \\
\text { representaciones, modelos, procedimientos, }\end{array}$ & $\begin{array}{l}\text {...el evaluado sopesa } \\
\text { procedimientos y } \\
\text { estrategias matemáticas } \\
\text { utilizadas para dar solución } \\
\text { a problemas planteados: } \\
\text { sostiene o refuta la }\end{array}$ \\
\hline
\end{tabular}


resultados, etc.) a partir de consideraciones

o conceptualizaciones matemáticas.

Incluye, entre otras cosas, que frente a un

problema o argumento que involucre

información cuantitativa u objetos

matemáticos se propongan o identifiquen

razones válidas; se utilicen adecuadamente

ejemplos y contraejemplos; se distingan

hechos de supuestos; y se reconozcan

falacias.

Fuente: tomado de Ministerio de Educación (2016).

interpretación de cierta información; argumenta a

favor o en contra de un

procedimiento de

resolución; acepta o

rechaza la validez 0

pertinencia de una

solución propuesta.

\section{Fuente: tomado de Ministerio de Educación (2016).}

Nota: Esta tabla ha sido adaptado del Módulo de Razonamiento cuantitativo Saber Pro.

\section{Método}

La presente investigación, cuyo objetivo es analizar las competencias matemáticas como factor de éxito para la realización de la Prueba Pro en universidades de Barranquilla, Colombia, se fundamentó en la metodología cuantitativa, pues en la investigación científica se requiere de un modelo conceptual que oriente la búsqueda de evidencias. Según Hernández et al. (2014), en estas "se usa la recolección de datos para probar hipótesis, con base en la medición numérica y el análisis estadístico para establecer patrones de comportamiento y probar teorías" (p. 4).

Asimismo, se enmarcó en un diseño de carácter no experimental transeccional, y su alcance es descriptivo, ya que "busca especificar propiedades y características importantes de cualquier fenómeno que se analice. Describe tendencias de un grupo o población" (Hernández et al, 2014, p. 92).

Es decir, se trata de estudios donde no se varía en forma intencional las variables independientes para ver su efecto sobre otras. En este tipo de investigación no experimental se observan los fenómenos tal como se dan en su contexto natural, para posteriormente analizarlos.

En la selección muestral se implementaron criterios ajustados a las posibilidades del investigador en cuanto a tiempo, espacio y accesibilidad, en función del conocimiento que el investigador tiene sobre la población, la cual está constituida por estudiantes universitarios de los dos últimos semestres de los programas de Contaduría Pública, Administración de Empresas e Ingeniería de Sistemas, pertenecientes a tres universidades privadas. Este criterio es relevante en la determinación del número de unidades de análisis.

La muestra se seleccionó mediante la técnica de muestreo estratificado. Según Hernández y Otros (2014, p. 149), "El Muestreo probabilístico estratificado es en el que la población se divide en segmentos y se selecciona una muestra para cada segmento". En este estudio se seleccionó en función de las disciplinas de estudio.

Es por ello, que en razón que la población es finita y representativa, se tomó el 100\%, ajustado al fundamento teórico aportado. Arias (2016, p. 82) lo define como toda "agrupación en la que se conoce la cantidad de unidades que la integran". Cabe destacar que, para efecto 
del presente aporte científico, se preservara el nombre de las instituciones universitarias seleccionadas. Las mismas aportaron un total de 244 participantes distribuidos de la siguiente forma:

Tabla 3.

Población

\begin{tabular}{l|l}
\hline Institución & $\begin{array}{l}\text { Unidades } \\
\text { análisis }\end{array}$ \\
\hline$A$ & 91 \\
$B$ & 83 \\
$C$ & 70 \\
\hline
\end{tabular}

Fuente: elaborado por los autores a partir de la muestra (2019).

Los datos se recolectaron por medio de un cuestionario definido por "un documento estructurado o no, que contiene un conjunto de reactivos (relativos a los indicadores de las variables) y 5 alternativas de respuestas", Siempre, Casi Siempre, Algunas Veces, Casi Nunca y Nunca (Chávez, 2007, p. 173).

En cuanto a la varianza, como se tomó toda la población la fórmula es:

$$
\sigma^{2}=\frac{\sum\left(x_{i}-\bar{x}\right)^{2}}{N}
$$

La desviación estándar es $\sigma=\sqrt{\sigma^{2}}$, siendo esta igual a 1,26.

\section{Resultados}

Aplicados los instrumentos, los datos recabados se procesaron en el programa Excel y los resultados se representaron por medio de cuadros o tablas. A continuación, se expone la información estadística en relación con la variable de estudio, enmarcada por el objetivo general: analizar las competencias matemáticas como factor de éxito para la realización de la Prueba Saber Pro en las Universidades de Colombia. Es por ello, que para describir las competencias matemáticas de los estudiantes, al momento de realizar la prueba Saber Pro; se analizaron las dimensiones competencias genéricas y razonamiento cuantitativo, correspondientes a la variable Competencias matemáticas; estas se traducen en indicadores como: conocimientos numéricos, producción de mensajes numéricos, nociones geométricas, interpretación y representación de datos, formulación y ejecución de competencias y argumentación de competencias. Los resultados de la Tabla 4 visualizan la media aritmética derivada de cada ítem, indicador y dimensión. Cabe destacar que los resultados reflejan la percepción expuesta por los estudiantes en cuanto a las competencias genéricas en el estudio de la matemática impulsada por sus docentes, y estos reflejan los siguientes datos en referencia a la dimensión Competencias genéricas.

Tabla 4

Variable: Competencias Matemáticas. Dimensión: competencias genéricas 


\begin{tabular}{|c|c|c|c|c|c|c|c|c|c|}
\hline & \multicolumn{3}{|c|}{ Numéricos } & \multicolumn{3}{|c|}{ numéricos } & \multicolumn{3}{|c|}{ Geométricas } \\
\hline Items & $\mathrm{I} 28$ & $\mathrm{I} 29$ & I30 & I31 & I32 & I33 & I34 & I35 & I36 \\
\hline $\begin{array}{l}\text { Media Por } \\
\text { Items }\end{array}$ & 3,40 & 3,80 & 3,30 & 2,70 & 3,40 & 4,10 & 3,50 & 3,55 & 3,60 \\
\hline $\begin{array}{l}\text { Media del } \\
\text { Indicador }\end{array}$ & \multicolumn{3}{|c|}{3,60} & \multicolumn{3}{|c|}{3,40} & \multicolumn{3}{|c|}{3,55} \\
\hline $\begin{array}{l}\text { Cierre del } \\
\text { Indicador }\end{array}$ & \multicolumn{3}{|c|}{ Casi siempre aplica } & \multicolumn{3}{|c|}{ Casi siempre aplica } & \multicolumn{3}{|c|}{ Casi siempre aplica } \\
\hline $\begin{array}{l}\text { Media de la } \\
\text { Dimensión }\end{array}$ & \multicolumn{9}{|c|}{3,52} \\
\hline $\begin{array}{l}\text { Cierre de la } \\
\text { Dimensión }\end{array}$ & \multicolumn{9}{|c|}{ Casi siempre aplica } \\
\hline Interpretación & \multicolumn{9}{|c|}{ La variante analizada se encuentra con una alta frecuencia } \\
\hline
\end{tabular}

Fuente: elaborado por los autores (2019).

Nota: Análisis realizado a partir de los datos recopilados en la fuente primaria.

En consecuencia, la Tabla 4 indica que los estudiantes encuestados consideran que la estrategia más promovida por lo docentes es la de "conocimientos numéricos", la cual alcanzó una Media Aritmética (MA) de 3, 60; seguida de "nociones geométricas", que arrojó un resultado 3,55 (MA); mientras que "producción de mensajes numéricos", que obtuvo 3,40, (MA), se constituiría en la estrategia con menor implementación, de acuerdo con la opinión de las unidades informativas entrevistadas. Sin embargo, todos los indicadores señalados se ubicaron en el rango "casi siempre", lo que deriva lógicamente en que la dimensión se ubique en el referido rango.

Tabla 5

Variable: Competencias Matemáticas. Dimensión: competencias genéricas

\begin{tabular}{|c|c|c|c|c|c|c|c|c|c|}
\hline \multicolumn{10}{|c|}{ Competencias genéricas } \\
\hline \multirow{2}{*}{$\begin{array}{c}\text { Indicadores } \\
\text { Ítems }\end{array}$} & \multicolumn{3}{|c|}{$\begin{array}{l}\text { Conocimientos } \\
\text { Numéricos }\end{array}$} & \multicolumn{3}{|c|}{$\begin{array}{l}\text { Producción de mensajes } \\
\text { numéricos }\end{array}$} & \multicolumn{3}{|c|}{$\begin{array}{l}\text { Nociones } \\
\text { Geométricas }\end{array}$} \\
\hline & $\mathrm{I} 37$ & I38 & I39 & $\mathrm{I} 40$ & I41 & I42 & I43 & $\mathrm{I} 44$ & I45 \\
\hline $\begin{array}{l}\text { Media Por } \\
\text { Ítems }\end{array}$ & 3,5 & 3,60 & 3,50 & 3.60 & 3,80 & 3.60 & 2,90 & 3,80 & 4,10 \\
\hline $\begin{array}{l}\text { Media del } \\
\text { Indicador }\end{array}$ & \multicolumn{3}{|c|}{3,53} & \multicolumn{3}{|c|}{3,67} & \multicolumn{3}{|c|}{3,60} \\
\hline $\begin{array}{l}\text { Cierre del } \\
\text { Indicador }\end{array}$ & \multicolumn{3}{|c|}{ Casi siempre aplica } & \multicolumn{3}{|c|}{ Casi siempre aplica } & \multicolumn{3}{|c|}{ Casi siempre aplica } \\
\hline $\begin{array}{l}\text { Media de la } \\
\text { Dimensión }\end{array}$ & \multicolumn{9}{|c|}{3,60} \\
\hline $\begin{array}{l}\text { Cierre de la } \\
\text { Dimensión }\end{array}$ & \multicolumn{9}{|c|}{ Casi siempre aplica } \\
\hline Interpretación & \multicolumn{9}{|c|}{ La variante analizada se encuentra con una alta frecuencia } \\
\hline
\end{tabular}

Por su parte, la Tabla 5 evidencia que los estudiantes universitarios de las carreras de Contaduría Pública, Administración de Empresas e Ingeniería de Sistemas piensan que la 
estrategia más impulsada por los docentes para el fortalecimiento del "razonamiento cuantitativo" correspondientes la de "formulación y ejecución", la cual obtuvo un valor Media Aritmética (MA) de 3, 67; seguida por el indicador "argumentación", que arrojó un resultado de 3,60 (MA); en tanto que el tercer lugar fue el indicador "interpretación y representación", que alcanzó 3,40 (MA). Por consiguiente, esta última es la estrategia con menor aplicación, de acuerdo con la percepción de los informantes entrevistados. Sin embargo, todos los indicadores señalados se ubicaron en el rango "casi siempre", por tanto la dimensión se ubicó en el rango señalado con 3,60 (MA).

Es importante plantear las siguientes inferencias: las medias aritméticas obtenidas por todos los indicadores se conservaron dentro del rango de "casi siempre" y la diferencia entre estas, no es representativa. En consecuencia, se infiere que las estrategias de enseñanza aplicada para promover los indicadores señalados como habilidades a ser aprendidas en el fortalecimiento de las competencias matemáticas a los estudiantes universitarios que hicieron parte de la población seleccionada, evidencia un equilibrio favorable en función de alcanzar los objetivos previstos.

\section{Tabla 6}

Variable: Competencias Matemáticas

\begin{tabular}{cc}
\hline \multicolumn{2}{l}{ Competencias Matemáticas } \\
\hline Competencias Genéricas & Razonamiento Cuantitativo \\
\hline 3,52 & 2,60 \\
\hline Media Variable \\
\hline 3,54 \\
\hline Moda \\
\hline 4 \\
\hline Mediana \\
\hline 4 \\
\hline Desviación Standart \\
1,26
\end{tabular}

Fuente: elaborado por los autores (2019).

Finalmente, se presenta la Tabla 6, en la cual se muestran las medidas de tendencia central, generadas de los datos recabados. En ella se evidencia que la respuesta con mayor frecuencia fue la número 4, correspondiente al rango de favorable. Asimismo, la mitad de los entrevistados se encuentra ubicados en el valor numérico 4, lo que indica que la mitad de los estudiantes consultados seleccionó como respuesta las dos opciones de tendencia positiva, es decir, "siempre" y "casi siempre". Sin embargo, la media aritmética de la variable "competencias matemáticas" arrojó un valor de 3, 54, (alcanzando el rango de "casi siempre"), desviados en promedio del referido dato en 1,26, según desviación estándar. Esto permite inferir que, pese a la desviación evidenciada, las respuestas en general pueden considerase en el sector de la favorabilidad. Cabe destacar que solo 12 encuestados respondió "nunca" a 
los ítems correspondientes a la dimensión "competencias genéricas" y solo tres a la dimensión "razonamiento cuantitativo", observándose una tendencia hacia valores medios o elevados de la escala planteada.

\section{Discusión y Conclusión}

Las competencias se definen como un conjunto de conocimientos, habilidades, actitudes, comprensiones y disposiciones cognitivas, socio-afectivas y psicomotoras apropiadamente relacionadas entre sí para facilitar el desempeño flexible, eficaz y con sentido de una actividad en contexto. En este sentido, de la investigación pueden resultar estrategias de enseñanza para promover competencias matemáticas en los estudiantes al momento de realizar la prueba Saber Pro. Previamente se deben considerar, sin embargo, las siguientes conclusiones:

El análisis de los datos recabados, así como la comparación de éstos con la teoría estudiada, reafirman la definición proporcionada por los teóricos consultados, los cuales coinciden en que las competencias matemáticas forman un conjunto de habilidades, así como destrezas, cuyo aprendizaje trasciende el ámbito académico, para constituirse en conocimiento imprescindible para la vida, y cuya aplicabilidad es necesaria para la realización desde actividades humanas simples hasta la consecución de grandes proyectos, sean individuales o colectivos.

En referencia al objetivo central del presente aporte científico, el cual estaba enfocado hacia analizar las competencias matemáticas como factor de éxito para la realización de la prueba Saber Pro en universidades de Barranquilla, Colombia, se concluyó que los estudiantes consultados consideran que las competencias matemáticas, así como sus dimensiones, competencias genéricas y razonamiento cuantitativo, son promovidas en los procesos de enseñanza "casi siempre". Este es un rango elevado, considerado como favorable.

Los resultados obtenidos muestran una tendencia central, pues son cercanos en cuanto a valor numérico, lo que permitió inferir que los indicadores son precisados por los estudiantes encuestados y considerados como una herramienta relevante en la elaboración de las pruebas Saber Pro, pero también para sus vidas académicas y profesionales.

Con relación al objetivo, el mismo se puede orientar al diseño de estrategias de enseñanza para promover competencias matemáticas en los estudiantes al momento de realizar la prueba Saber Pro.

\section{Referencias}

Aguilar, A. (2017). Crítica desde el pensamiento complejo a los métodos cuantitativos para el análisis educativo. El caso de los rendimientos educativos. Educación y Humanismo, 19(33), 357-368. Doi: https://doi.org/10.17081/eduhum.19.33.2649

Albrecht, E. Jiménez G. \& Jiménez, J. (2014). Estrategia Didáctica para Desarrollar la competencia "Comunicación y Representación" en Matemática. Escenarios, 12(1), 1733. Recuperado de https://docplayer.es/16472416-Estrategia-didactica-para- 
desarrollar-la-competencia-comunicacion-y-representacion-en-matematica.html

Arias, F. (2016). El proyecto de Investigación. Introducción a la metodología científica. Venezuela: Editorial Episteme.

Aristizabal, C. (2012). Aprendizaje Basado en Proyectos (A.B.Pr) Como estrategia de Enseñanza y Aprendizaje en la Educación Básica y Media. (Tesis de maestría, Universidad Nacional de Colombia, Bogotá). Recuperado de http://www.bdigital.unal.edu.co/9212/1/43253404.2013.pdf

Ausubel, D., Novak, J., \& Hanesian, H. (1983) Psicología Educativa: Un punto de vista cognoscitivo. México: Editorial Trillas.

Bambozzi, E \& Vadori, G. (2009). Competencias Genéricas. Colección Cuadernos de Investigación. Argentina: Universitaria de la Universidad de Villa María.

Bogisic, B., Bressan, A \& Crego, K. (2005). Razones para ensenar Geometría en la Educación Básica. Buenos Aires, Argentina: Editorial Novedades Educativas.

Bruno, A. (2000). Sentido numérico. Las matemáticas del siglo XX. Madrid, España: Libros y Ediciones Nivola.

Chávez, A. (2007). Introducción a la investigación educativa. Venezuela: Editorial Gráfica González.

Córdova, R. \& Oliveros, S. (2014). La Matemática Superior y las Competencias "Estrategia De Implementación de Competencias Matemáticas". Revista Gaceta Sansana, 1(4), 55-66. Recuperado de http://publicaciones.usm.edu.ec/index.php/GS/article/view/45/74

Fernández, J. (Agosto, 2010). Estrategias para desarrollar el cerebro matemático. Trabajo presentado en el Congreso Mundial De Neuroeducaciónasedh -Cerebrum, Lima, Perú. Recuperado de https://mafiadoc.com/estrategias-para-desarrollar-el-cerebromatematico-cpnsrosario-_5a2276001723dda07d8f9fc6.html

García, J. (2014). Ingeniería, Informática y Competencias. Revista Electrónica "Actualidades Investigativas en Educación, 14(1), 1-29. Recuperado de: https://www.redalyc.org/pdf/447/44729876015.pdf

Gutiérrez, L., Martínez, E. \& Nebreda S. (2008). Las competencias básicas en las áreas de Matemáticas. Cuadernos de educación de Cantabria, (5), 1-17. Recuperado de http://2633518-0.web-

hosting.es/blog/didact_mate/1.Competencias\%20b\%C3\%A1sicas\%20en\%20el\%20cu rr\%C3\%ADculo.pdf

Guzmán, C., Obonaga, G. \& Gutiérrez, O. (Mayo, 2015). Competencias matemáticas, diseño y selección de tareas para el aprendizaje de las matemáticas en ingeniería. Presentado en la XIV Conferencia interamericana de educación matemática, CIAEM, Chiapas, México. Recuperado de: http://xiv.ciaemredumate.org/index.php/xiv_ciaem/xiv_ciaem/paper/viewFile/246/138

Hernández, R., Fernández, C \& Baptista P (2014). Metodología de la Investigación. México: Graw Hill Education.

Instituto Colombiano para la evaluación de la educación (ICFES). (2013). Sistema Nacional de Educación Estandarizada de la educación. Alineación del Examen Saber 110. Recuperado de https://es.calameo.com/read/004256108acd8feb6fcdf 
Instituto Colombiano para la Evaluación de la Educación (ICFES). (2016) Módulo de Razonamiento cuantitativo Saber Pro 2016-2. Modulo: Razonamiento cuantitativo. Recuperado https://www.academia.edu/34141185/M\%C3\%B3dulo_de_Razonamiento_cuantitativ o_Saber_Pro_2016-2?auto=download

Íñiguez, P. (2015). El desarrollo de la competencia matemática en el aula de ciencias experimentales. Revista Iberoamericana de Educación, 67(2), 117-130. Doi: https://doi.org/10.35362/rie672256

Martínez, J. (2009). El lenguaje matemático; objetos matemáticos. Tema 1. Universidad de Alcalá de Henares. Recuperado de http://www2.uah.es/jmmartinezmediano/mate0/CIM\%20Tema\%201\%2001\%20Leng uaje\%20y\%20objetos\%20matemaicos.pdf

Méndez, P. (2013). Enseñanza inteligente de la matemática. Un modelo para promover las habilidades del pensamiento en el desarrollo lógico matemático. (Tesis Doctoral). Universidad Privada Dr. Rafael Belloso Chacín, Maracaibo, Venezuela.

Ministerio de Educación Nacional. (1998). Competencias Básicas en Matemáticas. Lineamientos Curriculares. Recuperado de http://www.mineducacion.gov.co/1621/articles16042_archivo_pdf2.pdf

Ministerio de Educación Nacional. (2005). Estándares básicos de competencias en lenguaje, matemáticas, ciencias y ciudadanas. Recuperado de http://bibliotecadigital.magisterio.co/libro/est-ndares-b-sicos-de-competencias-enlenguaje-matem-ticas-ciencias-y-ciudadanas

Ministerio de Educación Nacional. (2009). Competencias genéricas en educación superior. Recuperado de https://www.mineducacion.gov.co/1621/articles92779_archivo_pdf_Boletin13.pdf

OECD. (2005). The definition and selection of key competencies. Executive Summary. Recuperado de: http://www.pisa.oecd.org/dataoecd/47/61/35070367.pdf

Tobón, S. (2013). Formación integral y competencias. Pensamiento complejo, currículo, didáctica y evaluación. Colombia: ECOE Ediciones.

Vergara, J., Fontalvo, J., Muñoz, A. \& Valbuena, S. (2015). Estrategia didáctica para el fortalecimiento del razonamiento cuantitativo mediante el uso de las TIC. Revista del programa de matemáticas, 2(2), 71- $80 . \quad$ Recuperado de http://investigaciones. uniatlantico.edu.co/revistas/index.php/MATUA/article/view/140 $7 / 1064$

Villalonga, P. (2017) La competencia matemática. Caracterización de actividades de aprendizaje y de evaluación en la resolución de problemas en la enseñanza obligatoria. (Tesis Doctoral, Universidad Autónoma de Barcelona). Recuperado de https://www.tdx.cat/bitstream/handle/10803/457718/jmvp1de1.pdf?sequence=1\&isA llowed $=\mathrm{y}$ 